\title{
Clinical, epidemiological and virological features of acute hepatitis B in Italy
}

\author{
Ornella Zuccaro $\cdot$ Luisa Romanò $\cdot$ Alfonso Mele • Andrea Mariano • \\ Massimo Clementi · Maria Elena Tosti · Gloria Taliani · Claudio Galli • \\ Alessandro Remo Zanetti $\cdot$ Enea Spada $\cdot$ Study Group
}

Received: 20 October 2014 / Accepted: 6 February 2015 / Published online: 20 February 2015

(C) Springer-Verlag Berlin Heidelberg 2015

\begin{abstract}
Purpose To evaluate the association of hepatitis $\mathrm{B}$ virus (HBV) genotypes, basal core promoter (BCP)/precore (PC) and $\mathrm{S}$ gene mutations with the clinical-epidemiological characteristics of acute hepatitis B (AHB) in Italy.

Methods During July 2005-January 2007, 103 symptomatic AHB patients were enrolled and prospectively followed up at 15 national hospitals. HBV genotypes, BCP/ $\mathrm{PC}$ and $\mathrm{S}$ gene variants were determined by nested-PCR and direct sequence analysis.

Results Genotype D, A and F were detected in 49, 45 and $6 \%$ of patients, respectively. BCP, PC, and BCP plus PC variants were found in 3.1, 11.3 and $7.2 \%$ of patients, respectively. At enrollment, $68.3 \%$ of patients were hepatitis $\mathrm{B}$ e antigen ( $\mathrm{HBeAg}$ )-positive and $31.7 \%$ $\mathrm{HBeAg-negative.} \mathrm{BCP/PC} \mathrm{mutations} \mathrm{were} \mathrm{more} \mathrm{com-}$ mon in $\mathrm{HBeAg}$-negative than in $\mathrm{HBeAg}$-positive patients $(p<0.0001)$. Compared to genotype $\mathrm{D}$ patients, those
\end{abstract}

Ornella Zuccaro and Luisa Romanò contributed equally to this work.

Members of the Study Group are listed in the Appendix.

O. Zuccaro $\cdot$ A. Mele $\cdot$ M. E. Tosti

Surveillance and Health Promotion, National Center

of Epidemiology, Istituto Superiore di Sanità, Rome, Italy

L. Romanò · A. R. Zanetti

Department of Biomedical Sciences for Health, University

of Milan, Milan, Italy

\section{A. Mariano}

Clinical Research Department, National Institute for Infectious Diseases Lazzaro Spallanzani, Rome, Italy

M. Clementi

Laboratory of Microbiology and Virology, IRCCS San Raffaele,

Milan, Italy harboring non-D genotypes were more frequently males $(p=0.023)$, HBeAg-positive $(p<0.001)$, had higher bilirubin $(p=0.014)$ and viremia $(p=0.034)$ levels and less frequently carried BCP/PC mutations $(p<0.001)$. Non-D genotype patients more often were from Central Italy $(p=0.001)$ and reported risky sexual exposure $(p=0.021)$. Two patients had received vaccination before AHB: one harbored genotype F; the other showed a $\mathrm{S}$ gene mutation. Four patients developed fulminant AHB; mutations were found in 2 of 3 patients who underwent $\mathrm{BCP} /$ PC sequencing. After a 6-month follow-up, only $2(2.8 \%)$ patients developed persistent infection.

Conclusion AHB by non-D genotypes is increasing in Italy and is associated with risky sexual exposure. The ability of some genotypes to cause persistent and/or severe infection in Italy warrants larger studies for clarification.

Keywords Acute infection - Epidemiology - Genotypes · Hepatitis B $\cdot$ Mutations $\cdot$ Risk factors

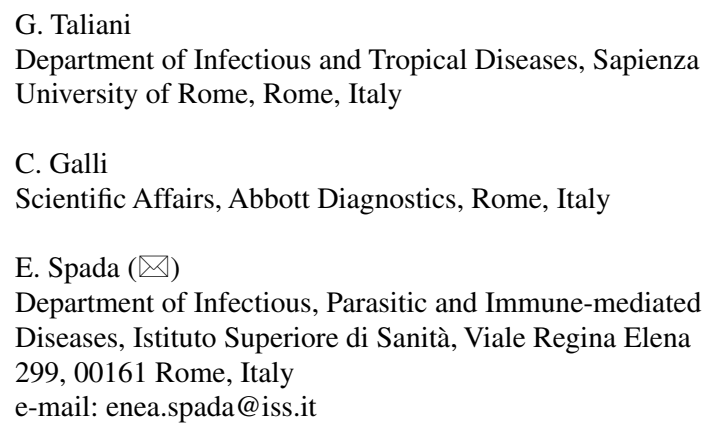




\section{Introduction}

Hepatitis B virus (HBV) infection is a major public health problem worldwide [1]. More than 240 million people have chronic infection and about 600,000 people die every year due to the consequences of acute or chronic hepatitis B [2].

The epidemiology of HBV infection in Italy has changed substantially over the last few decades and many cross-sectional and incidence studies have shown that Italy has reached a low level of endemicity [3-6]. Due to implementation of universal vaccination in 1991, all Italians aged 0 through 32 years were virtually protected against HBV infection in 2013. High-risk sexual behavior, cohabitation with a hepatitis B surface antigen (HBsAg)-positive carrier, healthcare-related exposures and several beauty treatments (including tattooing and piercing) remain the most frequent and important risk factors for acute hepatitis B (AHB) in Italy [6, 7]. However, it is conceivable that in Italy the clinical and epidemiological picture of HBV infection, as well as its current level of HBV endemicity, could change because of the increasing migration waves from highly endemic countries $[8,9]$.

The HBV genome is characterized by genetic heterogeneity. Ten genotypes (A to J) and a number of subtypes have been identified so far [10-12]. Interestingly, geographic distribution, clinical features, disease outcome, and response to therapy have been shown to differ according to HBV genotypes and subtypes in patients with acute and chronic hepatitis B (CHB) [1, 10-12]. Besides, different genotypes have also a distinct pattern of mutations in some HBV genomic regions [PreS and $\mathrm{S}$, basal core promoter $(\mathrm{BCP})$ and precore (PC), polymerase], which, in turn, are correlated with the clinical characteristics and outcome of both AHB and CHB $[10,12]$. BCP/PC mutations affecting hepatitis $\mathrm{B}$ e antigen ( $\mathrm{HBeAg}$ ) production have been widely investigated and have been found to be associated with a more severe and progressive form of chronic liver disease in patients with genotype $\mathrm{C}$ and D and with a fulminant course of AHB [10-13].

Genotype D, which is dominant in the Mediterranean basin, has been responsible for the vast majority of cases of acute and chronic hepatitis B in Italy for decades [14]. However, only a limited number of Italian studies have focused on the relevance of different HBV genotypes and mutations for the natural history of both AHB and CHB [14-20]. Particularly as regards the natural history of AHB, so far only three Italian studies have investigated the influence of HBV genotypes [17-19], and only one has taken into consideration the role of genome mutations deemed able to influence the clinical disease course [19].

In this prospective study we enrolled a cohort of patients with AHB and evaluated the natural course of the infection by analyzing epidemiological, clinical, serological and virological characteristics, including HBV genotype and mutations in S gene and BPC/PC regions. This investigation was also useful to evaluate the impact of immigration on the introduction and spread in Italy of unusual and clinically relevant genotypes and mutations $[8,9]$.

\section{Patients and methods}

Participants and setting

This prospective cohort study was conducted at 15 Infectious Disease Units scattered throughout Italy. Between July 2005 and January 2007, all consecutive patients with symptomatic AHB were enrolled in the study after obtaining written informed consent.

Mandatory inclusion criteria were: high titer [sample/ cutoff $(\mathrm{S} / \mathrm{CO})$ ratio $\geq 10]$ of immunoglobulin $\mathrm{M}(\mathrm{IgM})$ antibodies to hepatitis B core antigen (anti-HBc) [21]; significant increase in serum alanine aminotransferase (ALT) levels (>10 times the upper limit of normal); acute illness compatible with viral hepatitis. Patients positive for IgM antibodies to hepatitis A virus (HAV) or with any other possible cause of acute liver damage were excluded. All available medical records of patients enrolled in the study were reviewed to ascertain the absence of positive $\mathrm{HBsAg}$ or anti-HBc tests before hospital admission. Immunization records of patients who reported previous HBV vaccination were checked to verify the completeness and correctness of vaccination schedule.

A questionnaire recording information on demographic characteristics and risk factors for AHB was administered to all patients. All patients underwent clinical, biochemical and virological assessment at enrolment, then weekly during the first month and, in the event of persistence of HBsAg in serum, every 3 months thereafter for a total of 12 months or until the patients withdrew their participation. Patients were considered to have recovered from AHB, if steady normalization of serum aspartate aminotransferase (AST) and ALT levels together with HBsAg clearance occurred within 6 months after disease onset. Persistence of HBsAg in serum for more than 6 months was considered evidence of chronic disease evolution. Patients were considered to have developed fulminant hepatitis if they showed porto-systemic encephalopathy and coagulopathy (reduction in prothrombin activity below $35 \%$ ) within 8 weeks after disease onset.

All patients gave written informed consent before entering the study. The study was approved by the Ethics Committee of the Istituto Superiore di Sanità.

\section{Serological tests}

All patients were tested for HBsAg, anti-HBc IgM, antiHAV IgM, anti-hepatitis C virus (HCV), anti-hepatitis 
delta virus (HDV) and anti-human immunodeficiency virus (HIV) antibodies by commercial immunoassays at the enrolling clinical sites. Further serological characterization of HBV infection was performed at the reference laboratory by commercial chemiluminescent assays on the ARCHITECT platform (Abbott Diagnostics, Wiesbaden, Germany). Briefly, quantitative methods were used for HBsAg (sensitivity of $0.05 \mathrm{IU} / \mathrm{mL}$ ) and anti-HBs (upper quantitation limit of $1000 \mathrm{mIU} / \mathrm{mL}$ ); total anti-HBc, $\mathrm{HBeAg}$, anti-HBe were determined by qualitative assays, while anti-HBc IgM was examined by a semiquantitative assay.

\section{HBV DNA detection and characterization}

HBV DNA was assayed in serum by quantitative PCR (Cobas TaqMan HBV Test, Roche Molecular Systems, USA) at enrolment, a week later and then-in case of positivity-until two consecutive samples collected according to the follow-up schedule tested negative. HBV genotypes, $\mathrm{BCP} / \mathrm{PC}$ and $\mathrm{S}$ gene variants were determined on the first HBV DNA-positive sample by nested-PCR in the core/ precore and $\mathrm{S}$ regions, followed by direct sequence analysis of the amplicons, as previously reported [22,23].

\section{Statistical analysis}

Mann-Whitney test was used for continuous variables to assess differences between distributions. Pearson's Chisquared test or Fisher's exact test was used for comparison of frequencies between groups. A $p$ value of 0.05 was considered significant. All statistical analyses were performed using STATA, version 11.2 (StataCorp LP, College Station, TX).

\section{Results}

General clinical-epidemiological and virological characteristics of AHB patients at enrolment

A total of 103 consecutive AHB patients were enrolled in the study (Table 1). All patients were symptomatic and $97(94.2 \%)$ of them had jaundice. All patients showed increased ALT serum levels at least ten times the upper limit of normal and had high-titer anti-HBc IgM antibodies [average sample/cutoff $(\mathrm{S} / \mathrm{CO})$ ratio $32.09 \pm 9.24$ ]; all but two patients were $\mathrm{HBsAg}$-positive. $\mathrm{HBeAg}$ and anti-HBe were tested in 101 patients. Of them, $52(51.5 \%)$ were HBeAg-positive/anti-HBe-negative, 29 (28.7\%) HBeAgnegative/anti-HBe-positive, 17 (16.8\%) HBeAg-positive/ anti-HBe-positive and $3(2.9 \%)$ HBeAg-negative/antiHBe-negative (Table 1; Fig. 1a). High-risk sexual behavior and parenteral exposures unrelated to drug use (e.g., nosocomial exposure, cosmetic treatment with percutaneous exposure and cohabitation with a HBsAg-positive carrier) were the most frequently reported risk factors (Table 1).

A concomitant infection with HCV, HDV and HIV was detected in 4/102 (3.9\%), 1/74 (1.4\%) and 4/74 (5.4\%) patients who were tested for these viruses (Table 1).

Serum HBV DNA was examined at disease onset in 102 patients, of whom 101 tested positive (99\%); one was already negative. HBV genotype was determined in 100 of the 101 viremic patients: 49 harbored genotype $\mathrm{D}, 45$ genotype $\mathrm{A}$ and six genotype $\mathrm{F}$ (Table 1; Fig. 1b).

$\mathrm{BCP} / \mathrm{PC}$ variants could be determined in 97 of the 101 HBV DNA-positive patients. Either BCP (A1762T/ $\mathrm{G} 1764 \mathrm{~A})$ or PC (G1896A) variants were found in $3(3.1 \%)$ and $11(11.3 \%)$ of these patients, respectively; the simultaneous presence of BCP and PC mutations was observed in 7 (7.2\%) patients while the remaining $76(78.4 \%)$ patients harbored BCP/PC wild-type sequences (Table 1; Fig. 1c).

$\mathrm{HBe} \mathrm{Ag} / \mathrm{anti}-\mathrm{HBe}$ status and $\mathrm{BCP} / \mathrm{PC}$ mutations were tested in 96 patients (Table 2). A significantly higher prevalence of $\mathrm{BCP} / \mathrm{PC}$ variants was observed in $\mathrm{HBeAg}$-negative patients compared to $\mathrm{HBeAg}$-positive patients (19/29 or 65.5 vs $2 / 67$ or $2.9 \%$; $p<0.001$ ).

The analysis of $\mathrm{S}$ gene region mutations was performed on the "a" determinant sequences from 91 patients, seven of whom $(7.7 \%)$ showed mutated sequences, for a total of 12 mutations: T126I, S132F, S143L; N131T $(n=2)$, $\mathrm{F} 134 \mathrm{Y} / \mathrm{N}(n=2$ each $)$ and T143 M/S/F.

Clinical-epidemiological and virological characteristics of AHB patients at enrolment according to HBV genotype

Data on both infecting genotype and HBe/anti-HBe status were available for 99 patients (Tables 2, 3). Patients infected with non-D genotypes were more often $\mathrm{HBeAg}$ positive (66.2 vs $33.8 \%)$ and less frequently HBeAg-negative (16.1 vs $83.9 \%$ ) than patients harboring genotype D (Table 2; $p<0.001$ ). The association between HBV genotype and $\mathrm{HBeAg}$ status was still statistically significant, even excluding from the analysis patients with $\mathrm{BCP} /$ PC mutations $(20 \mathrm{HBeAg}+/ 7 \mathrm{HBeAg}-$ in genotype $\mathrm{D}$ patients versus $45 \mathrm{HBeAg}+/ 3 \mathrm{HBeAg}-$ in genotype non-D patients, $p=0.030$ ). Patients infected with non-D genotypes were more often male $(p=0.023)$, had higher total bilirubin level $(p=0.014)$ and HBV viral load $(p=0.034)$ at enrolment than patients harboring genotype $\mathrm{D}$ (Table 3 ). Data on HBV genotype and mutations in the PC and BCP regions were available for 97 patients: the overall prevalence of $\mathrm{BCP} / \mathrm{PC}$ mutations was significantly higher in patients infected with genotype D than in those harboring non-D genotypes (19/46 or 41.3 vs $2 / 51$ or $3.9 \%$; $p<0.001$ ) (Table 3). 
Table 1 Baseline demographic and clinical characteristics in 103 patients with acute hepatitis B diagnosed at 15 Italian Infectious Disease Units (2005-2007)

\begin{tabular}{|c|c|}
\hline Gender (No. \%) & 103 \\
\hline Males & $70(68.0)$ \\
\hline Females & $33(32.0)$ \\
\hline Age, median years (range) & $37(16-80)$ \\
\hline Geographic area of residence (No. \%) & 103 \\
\hline North Italy & $23(22.3)$ \\
\hline Central Italy & $35(34.0)$ \\
\hline Southern Italy and islands & $45(43.7)$ \\
\hline Birth country (No. \%) & 103 \\
\hline Italy & $89(86.4)$ \\
\hline Abroad & $14(13.6)$ \\
\hline Years of schooling (No. \%) & 89 \\
\hline$\leq 8$ & $45(50.6)$ \\
\hline$\geq 9$ & $44(49.4)$ \\
\hline Risk factors $(\text { No. } \%)^{\mathrm{a}}$ & 103 \\
\hline Intravenous drug use & $6(5.8)$ \\
\hline Sexual exposure ${ }^{b}$ & $40(38.8)$ \\
\hline Other ${ }^{\mathrm{c}}$ & $25(24.3)$ \\
\hline Undetermined & $22(21.3)$ \\
\hline Jaundice (No. \%) & $97(94.8)$ \\
\hline Hospitalization (No. \%) & $102(99.0)$ \\
\hline AST (IU/ml), median (range) & $1836(321-5558)$ \\
\hline ALT (IU/ml), median (range) & $2431(851-6324)$ \\
\hline Total bilirubin $(\mathrm{mg} / \mathrm{dL})$, median (range) & $15(1.1-48.9)$ \\
\hline Fulminant hepatitis (No. \%) & $4(3.9)$ \\
\hline Deaths (No. \%) & $1(1.0)$ \\
\hline Transplantations (No. \%) & $2(2.2)$ \\
\hline HBV DNA-positive (No. \%) & $101(98.0)$ \\
\hline HBV DNA (copies/mL), median (range) & $110,683(7-150,000,000)$ \\
\hline anti-HBc IgM-positive & $103(100.0)$ \\
\hline HBsAg-positive (No. \%) & $101(98.0)$ \\
\hline HBeAg/anti-HBe status (No. \%) & 101 \\
\hline $\mathrm{HBeAg}+/$ anti-HBe- & $52(51.5)$ \\
\hline $\mathrm{HBeAg}-/$ anti-HBe+ & $29(28.7)$ \\
\hline $\mathrm{HBeAg}+/$ anti-HBe+ & $17(16.8)$ \\
\hline $\mathrm{HBeAg}-/$ anti-HBe- & $3(2.9)$ \\
\hline Coinfection with HCV (No. \%) & $4(3.9)$ \\
\hline Coinfection with HDV (No. \%) & $1(1.4)$ \\
\hline Coinfection with HIV (No. \%) & $4(5.4)$ \\
\hline HBV genotype (No. \%) & 100 \\
\hline A & $45(45.0)$ \\
\hline $\mathrm{D}$ & $49(49.0)$ \\
\hline $\mathrm{F}$ & $6(6.0)$ \\
\hline
\end{tabular}

Of the seven patients who showed mutated sequences in the "a" determinant of the $\mathrm{S}$ gene, three patients harbored genotype D and carried three mutations: T126I, S132F, S143L; while four patients harbored genotype $\mathrm{A}$ and
Table 1 continued

\begin{tabular}{ll}
\hline HBV core/precore mutations (No. \%) & 97 \\
Precore (PC) & $11(11.3)$ \\
Basal core promoter (BCP) & $3(3.1)$ \\
PC + BCP & $7(7.2)$ \\
Wild type & $76(78.4)$ \\
\hline a Cases may have more than one risk factor \\
b Promiscuous sexual activity (more than two sexual partners), and \\
unsafe (i.e., no or seldom condom use during occasional sexual inter- \\
course) homo-bisexual intercourses \\
c Cosmetic treatment with percutaneous exposure (piercing, tattoo- \\
ing, manicure/pedicure, barber shop shaving and acupuncture), living \\
with a HBsAg+ carrier, nosocomial exposure, dental treatment
\end{tabular}

carried nine mutations: N131T $(n=2), \mathrm{F} 134 \mathrm{Y} / \mathrm{N}(n=2$ each) and T143/M/S/F.

The majority of patients $(86.4 \%)$ were born and resided in Italy, including all those harboring genotype F. Fourteen patients were immigrants from eastern Europe (Romania [ $n=6$ ], Ukraine [4], Bulgaria and Poland) and northern Africa (Tunisia and Marocco). No significant difference in genotype distribution was detected according to patient's country of birth. Genotype D occurred more frequently in patients residing in Southern Italy and Islands as opposed to non-D genotypes, which were more frequent in patients from Central Italy ( $p=0.001$ ) (Table 3).

Interestingly, genotype D was more frequently detected in patients who reported intravenous drug use or other parenteral exposure, while patients reporting high-risk sexual behavior harbored non-D genotypes more often $(p=0.021)$ (Table 3). Overall, more than half of the patients harboring non-D genotypes reported high-risk sexual behavior, (4/6 or 66.7 and $21 / 45$ or $46.7 \%$ of genotype $\mathrm{F}$ and A patients, respectively). About $10 \%$ of AHB patients reported homobisexual intercourses in the 6 months before the disease onset; this exposure was particularly common among patients infected with non-D genotypes, notably genotype A. However, by comparing only genotype D and genotype A patients, the association between genotype A and highrisk sexual exposure resulted to be only marginally significant $(p=0.07)$.

AHB in previously vaccinated individuals

Two patients enrolled in this study had received a complete and well-documented full course of primary $\mathrm{HBV}$ vaccination several years before AHB occurrence, both of whom did not undergo pre- or post-vaccination serologic testing. The first patient, a 44-year-old man suffering from insulindependent diabetes mellitus since age 22, received HBV vaccination about 6 years before AHB presentation. At enrolment he was HBsAg-positive, anti-HBs-negative, HBeAg-positive/ anti-HBe-negative and harbored genotype $\mathrm{F}$. The second 


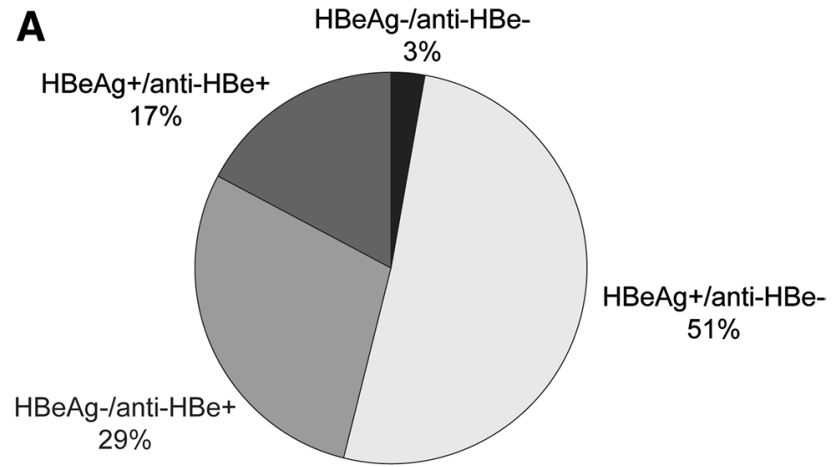

B
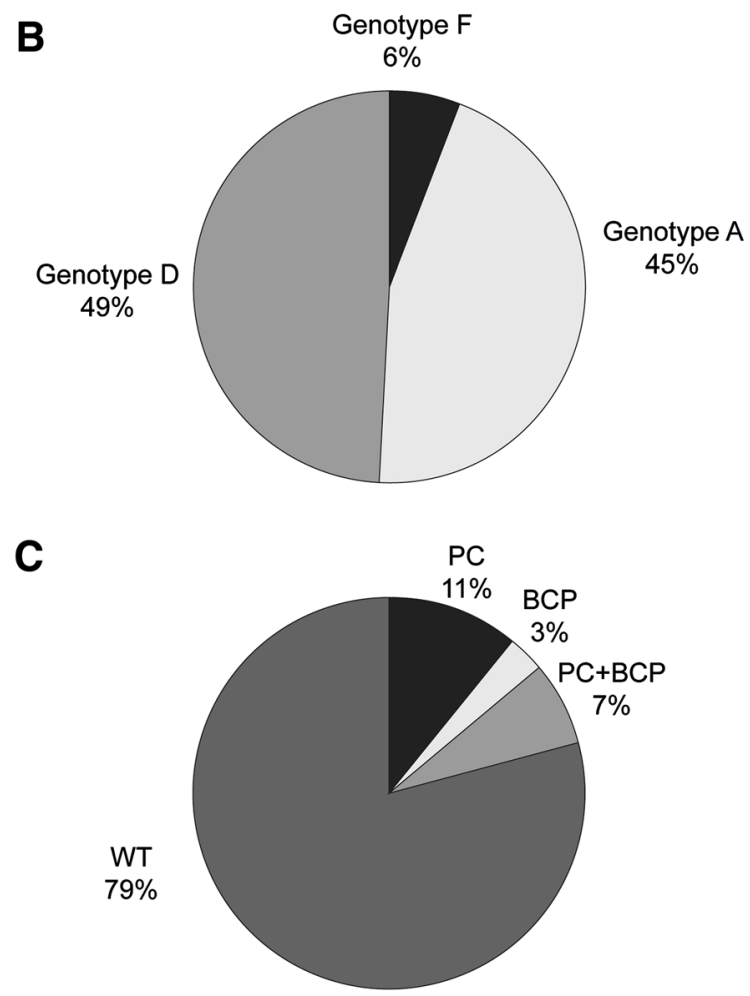

Fig. 1 Distribution of hepatitis B e antigen (HBeAg)/antibodies to hepatitis e antigen (anti-HBe) (a), genotypes (b) and basal core promoter (BCP)/precore (PC) mutations (c) among 103 consecutive patients with acute hepatitis B diagnosed at 15 Italian Infectious Disease Units between July 2005 and January 2007

patient, a 19-year-old man with a history of intravenous drug use, completed primary vaccination about 15 years before AHB presentation. At enrolment, this patient tested HBsAgpositive, anti-HBs-negative, HBeAg-negative/anti-HBe-positive. Besides, he harbored genotype D and showed the T126I mutation in the "a" determinant of the $\mathrm{S}$ gene.

\section{Clinical outcome of AHB}

Four AHB patients $(3.9 \%)$ developed fulminant hepatitis (Table 4). One patient died, two patients underwent transplantation and one other was transferred to a different hospital and lost to follow-up. BCP/PC mutations could be assessed for three of these four patients: one patient, infected with genotype $\mathrm{D}$, showed the simultaneous presence of BCP and PC variants; another one, infected with genotype $\mathrm{A}$, had a $\mathrm{BCP}$ mutation; and the last patient, harboring genotype $\mathrm{D}$, had wild-type sequences both in $\mathrm{BCP}$ and $\mathrm{PC}$ regions. No coinfection with $\mathrm{HCV}, \mathrm{HDV}$ and HIV was detected in these four patients and none of them reported history of chronic liver disease.

A follow-up of at least 6 months, suitable to establish AHB recovery or chronic evolution, was available for 72 (69.9\%) patients (37 harboring genotype D, 29 genotype $\mathrm{A}$, five genotype $\mathrm{F}$ and one with undetermined genotype). Persistence of HBsAg for more than 6 months was observed in two of 72 patients $(2.8 \%$; $95 \%$ confidence interval 0.3-10.0). Both patients were women and aged $\geq 50$ years. One of them was infected with genotype A, while the other with genotype D (Table 3 ). In both cases $\mathrm{BCP} / \mathrm{PC}$ wild-type sequences were found. Only one of the two patients was tested for anti-HIV antibodies and was found to be negative. Among the remaining 70 patients who recovered from AHB, 22 (31.4 \%) (18 with genotype $\mathrm{D}$ and four with genotype non-D), 23 (32.9\%) (six with genotype D and 16 with genotype non-D) and 25 (35.7 \%) patients (12 with genotype D and 13 with genotype nonD) cleared HBsAg within the third month, between 3 and 6 months, and at the sixth month after enrolment, respectively. Among these patients, $\mathrm{HBsAg}$ clearance occurred significantly earlier $(p=0.001)$ in those harboring genotype $\mathrm{D}$ than in those infected with non-D genotypes.

Thirty-one AHB patients were followed up for less than 6 months. Twenty-three of these patients withdrew from the study within the first month after enrolment; they were still HBsAg-positive at the last visit and none of them showed evident decrease ( $>2 \operatorname{logs}$ ) in HBsAg levels during follow-up. Six patients were followed up for a median period of 99.5 days (range 70-115) after enrolment and all of them, albeit still positive at the last visit, showed an evident decrease in HBsAg levels (median decrease 4.5 logs; range 4-6 logs) compared to baseline values. Finally, two patients, who withdrew from the study after 5 months of follow-up, were HBsAg-negative at the last visit.

No significant differences were found at enrolment between subjects followed for at least 6 months and those lost to follow-up regarding epidemiological, clinical, serological and virological characteristics (including HBV genotype distribution) (data not shown).

\section{Discussion}

In this study, we have provided an overall comprehensive description of the clinical, epidemiological and virological 
Table 2 Distribution of HBV genotypes and precore (PC)/basal core promoter (BCP) region mutations according to $\mathrm{HBeAg} / \mathrm{anti}-\mathrm{HBe}$ status in 103 patients with acute hepatitis B diagnosed at 15 Italian Infectious Disease Units (2005-2007)

\begin{tabular}{|c|c|c|c|c|c|c|}
\hline HBeAg/anti-Hbe status & $\begin{array}{l}\mathrm{PC} \text { and/or BCP (No. } \\
\%)\end{array}$ & Wild type & )Total (No. \%) & $\begin{array}{l}\text { Genotype D (No. } \\
\%)\end{array}$ & $\begin{array}{l}\text { Non-D genotype (No. } \\
\%)\end{array}$ & $\begin{array}{l}\text { Total (No. } \\
\%)\end{array}$ \\
\hline $\mathrm{HBeAg}+/$ anti-HBe- & $1(2.0)$ & $49(98.0)$ & $50(100.0)$ & $17(33.3)$ & $34(66.6)$ & $51(100.0)$ \\
\hline $\mathrm{HBeAg}-/$ anti-HBe+ & $18(66.6)$ & $9(33.3)$ & $27(100.0)$ & $24(82.8)$ & $5(17.2)$ & $29(100.0)$ \\
\hline $\mathrm{HBeAg}+/$ anti-HBe+ & $1(5.9)$ & $16(94.1)$ & $17(100.0)$ & $6(35.3)$ & $11(64.7)$ & $17(100.0)$ \\
\hline $\mathrm{HBeAg}$-/anti-HB- & $1(50.0)$ & $1(50.0)$ & $2(100.0)$ & $2(100.0)$ & $0(0.0)$ & $2(100.0)$ \\
\hline $\mathrm{HBeAg}+$ & $2(3.0)$ & $65(97.0)$ & $67(100.0)$ & $23(33.8)$ & $45(66.2)$ & $68(100.0)$ \\
\hline anti-HBe+ & $19(43.2)$ & $25(56.8)$ & $44(100.0)$ & $30(65.2)$ & $16(34.8)$ & $46(100.0)$ \\
\hline $\mathrm{HBeAg}-$ & $19(65.5)$ & $10(34.5)$ & $29(100.0)$ & $26(83.9)$ & $5(16.1)$ & $31(100.0)$ \\
\hline anti-HBe- & $2(3.8)$ & $50(96.2)$ & $52(100.0)$ & $19(35.8)$ & $34(64.2)$ & $53(100.0)$ \\
\hline
\end{tabular}

anti-HBe antibodies to hepatitis e antigen, $H B e A g$ hepatitis B e antigen, $H B V$ hepatitis B virus, $B C P$ basal core promoter, $P C$ precore

Table 3 Demographic, clinical, biochemical and virological characteristics according to HBV genotype in patients with acute hepatitis B diagnosed at 15 Italian Infectious Disease Units (2005-2007)

a Cases may have more than one risk factor

b Promiscuous sexual activity (more than two sexual partners during the previous 6 months), and unsafe (i.e., no or seldom condom use during occasional sexual intercourse) homobisexual intercourses

c Treatment with percutaneous exposure (piercing, tattooing, manicure/pedicure, barber shop shaving and acupuncture), living with a $\mathrm{HBsAg}+$ carrier, nosocomial exposure, dental treatment

\begin{tabular}{|c|c|c|c|}
\hline & $\begin{array}{l}\text { Genotype D } \\
\text { No. }(\%)\end{array}$ & $\begin{array}{l}\text { Genotype non-D } \\
\text { No. }(\%)\end{array}$ & $p$ value \\
\hline No. cases & 49 & 51 & \\
\hline Age (years), median (range) & $34(16-80)$ & $37(21-57)$ & 0.417 \\
\hline Males & $28(57.13)$ & $40(78.4)$ & 0.023 \\
\hline \multicolumn{4}{|l|}{ Geographic area of residence } \\
\hline North & $11(22.4)$ & $11(21.6)$ & \multirow[t]{3}{*}{0.001} \\
\hline Central & $9(18.4)$ & $26(51.0)$ & \\
\hline Southern and islands & $29(59.2)$ & $14(27.4)$ & \\
\hline \multicolumn{4}{|l|}{ Birth country } \\
\hline Italy & $39(79.6)$ & $47(92.2)$ & \multirow[t]{2}{*}{0.070} \\
\hline Abroad & $10(20.4)$ & $4(7.8)$ & \\
\hline \multicolumn{4}{|l|}{ Risk factors ${ }^{\mathrm{a}}$} \\
\hline Intravenous drug use & $5(12.5)$ & $1(2.6)$ & \multirow[t]{4}{*}{0.021} \\
\hline Sexual exposure ${ }^{b}$ & $14(35.0)$ & $25(64.1)$ & \\
\hline Other ${ }^{c}$ & $21(52.5)$ & $13(33.3)$ & \\
\hline Undetermined & $9(18.4)$ & $12(23.5)$ & \\
\hline Chronic evolution & $1(2.7)$ & $1(2.9)$ & 1.000 \\
\hline Fulminant hepatitis & $2(4.1)$ & $1(2.0)$ & 0.614 \\
\hline Deaths & $1(2.0)$ & $0(0.0)$ & 0.490 \\
\hline Transplantations & $0(0.0)$ & $1(2.0)$ & 1.000 \\
\hline ALT (IU/ml), median (range) & $2354(851-4974)$ & $2436(1110-6324)$ & 0.268 \\
\hline AST (IU/ml), median (range) & $1857.5(321-5558)$ & $1795(361-4230)$ & 0.909 \\
\hline Total bilirubin (mg/dL), median (range) & $13.65(1.1-32)$ & $17.1(1.6-48.9)$ & 0.014 \\
\hline \multicolumn{4}{|l|}{ Precore and basal core promoter variations } \\
\hline Wild type & $27(58.7)$ & $49(96.1)$ & \multirow[t]{2}{*}{$<0.001$} \\
\hline Mutant strain & $19(41.3)$ & $2(3.9)$ & \\
\hline HBV DNA (copies $/ \mathrm{mL}$ ), median (range) & $66,761\left(7-150 \times 10^{6}\right)$ & $332,485\left(31-150 \times 10^{6}\right)$ & 0.034 \\
\hline \multicolumn{4}{|l|}{$\mathrm{HBeAg} / \mathrm{anti}-\mathrm{HBe}$ status } \\
\hline $\mathrm{HBeAg}+/$ anti-HBe- & $17(33.3)$ & $34(66.6)$ & \multirow[t]{4}{*}{$<0.001$} \\
\hline $\mathrm{HBeAg}-/$ anti-HBe+ & $24(82.8)$ & $5(17.2)$ & \\
\hline $\mathrm{HBeAg}+/$ anti-HBe+ & $6(35.3)$ & $11(64.7)$ & \\
\hline $\mathrm{HBeAg}-/$ anti-HBe- & $2(100.0)$ & $0(0.0)$ & \\
\hline
\end{tabular}


Table 4 Clinical and laboratory data of four fulminant hepatitis B cases occurred among a cohort of 103 patients with acute hepatitis B diagnosed at 15 Italian Infectious Disease Units (2005-2007)

\begin{tabular}{|c|c|c|c|c|c|c|}
\hline Age & Sex & Outcome & HBV Genotype & $\begin{array}{l}\text { Precore }(\mathrm{PC}) / \mathrm{basal} \text { core } \\
\text { promoter }(\mathrm{BCP}) \text { variants }\end{array}$ & Risk factor & $\mathrm{HBeAg} /$ anti-HBe status \\
\hline 46 & M & Transplantation & Undetermined & Undetermined & Sexual exposure ${ }^{a}$ & $\mathrm{HBeAg}-/$ anti-HBe+ \\
\hline 21 & $\mathrm{~F}$ & Transplantation & A & $\mathrm{BCP}$ & Undetermined & $\mathrm{HBeAg}+/$ anti-HBe+ \\
\hline 30 & $\mathrm{~F}$ & Death & $\mathrm{D}$ & Wild type & Undetermined & $\mathrm{HBeAg}-/$ anti-HBe+ \\
\hline 45 & M & Loss to follow-up & $\mathrm{D}$ & PC, BCP & Sexual exposure ${ }^{\mathrm{a}}$ & Undetermined \\
\hline
\end{tabular}

a Promiscuous sexual activity (more than two sexual partners during the previous 6 months), and unsafe (i.e., no or seldom condom use during occasional sexual intercourse) homo-bisexual intercourses

scenario of AHB in Italy as it appeared many years after the occurrence of two events of great epidemiological and public health importance, both dated 1991: the introduction of universal hepatitis B vaccination and the beginning of massive immigration to Italy from countries with high HBV endemicity.

So far only three Italian studies, performed in Southern Italy, have investigated the influence of HBV genotypes on the clinical-epidemiological features of AHB [17-19] and only one of these studies (which focused on patients with severe AHB) has taken into consideration the role of genome mutations deemed able to influence the clinical disease course [19]. Besides, these studies were conducted in one or at most two towns of the same region and enrolled about $100-140$ patients during nearly 10 years. Thus, in these studies only the analysis of separate time intervals of recruitment (but with a smaller number of observations in each interval) allowed authors to point out major changes to the clinical-epidemiological and virological profiles of the disease.

A major finding of our study is that more than half (51\%) of the observed AHB cases were due to non-D genotypes, mainly genotype A and F. Genotype A is prevalent in North Europe, the USA, Sub-Saharan and Western Africa $[10,11]$, while genotype $\mathrm{F}$ is common in Central and South America, although it has already been observed in Italy $[10,11,14,17,19,24]$. The prevalence of genotype A $(45 \%)$ we found in our study was considerably higher than that reported in the same range time by Coppola et al. (13.7\%) [17] and by Scalia et al. (27 \%) [18]. However, these studies were performed in Southern Italy, where non-D genotypes, basing on our findings, are less frequently detected than genotype $\mathrm{D}$.

Thus, looking at our and other available data [17-20, 24], it seems that in Italy the frequency of AHB cases due to genotype A has increased over time, while AHB cases harboring other non-D genotypes have been detected only sporadically. This scenario is consistent with the results obtained in a large sample of CHB patients in North-Eastern Italy during the years 2002-2004; among these patients the prevalence of genotype D and A was 70.6 and $25 \%$, respectively, while other unusual genotypes $(\mathrm{C}, \mathrm{E}, \mathrm{F}, \mathrm{H})$ were detected in very low percentages, mainly in foreigners [14].

In our study, high-risk sexual exposure was the most frequently reported risk factor for AHB and it was significantly associated with infection by non-D genotypes. An association between infection with non-D genotypes (particularly genotype A) and high-risk sexual exposure was also observed in previous Italian studies $[17,18]$. The increasing in prevalence of genotype A over time among AHB patients and its association with high-risk sexual exposure have been well documented by several studies in Japan, where genotype $\mathrm{C}$ traditionally predominated [25-27]. The association between genotype A and highrisk sexual exposure has also been reported in studies conducted in some Northern European countries, where genotype A has always been the most prevalent [28, 29]. The above reported change in the molecular epidemiology pattern of AHB over time, well documented in Italy and Japan, has been mainly attributed to the immigration phenomenon $[17,18,25,26]$. It has been postulated that genotype A was firstly transmitted from infected immigrants to susceptible natives by sexual contact, and then it also spread among the native population, remaining, however, mainly confined to people with at risk sexual behavior [17, 25, 26].

The reasons for the correlation between genotype A and sexual transmission have not yet been fully elucidated. The high viral load that we and others found in genotype A infection [27, 30, 31] increases the HBV concentrations in semen and other body fluids of carriers, thus increasing the risk of transmission by sexual intercourse. Moreover, Japanese studies and our results have shown that genotype A infection tends to persist following AHB and this may further influence its spread [26, 27, 30, 31]. However, also genotype D can be efficiently transmitted through sexual contact: about one-third of our AHB patients harboring genotype D also reported high-risk sexual behavior. Thus, other epidemiological features, in addition to immigration, may have contributed to the current molecular epidemiological pattern of AHB in Italy. Indeed, most probably this pattern is the consequence of the important changes in 
HBV epidemiology that occurred throughout the last decades in Italy. These changes are mainly due to the universal vaccination against HBV that has gradually elevated the age of the susceptible population. At the time of the study, all Italians $\leq 27$ years were virtually protected against HBV. The shift in the age of the HBV susceptible population has produced a consequent change in the role of different risk factors, with a significant reduction in the role of intrafamilial contact and intravenous drug use and an increase in the role of unsafe homosexual and heterosexual exposure. This has produced, in turn, an increase in frequency of AHB cases due to non-D genotypes, which preferably spread among people with high-risk sexual behavior. Studies based on phylogenetic analysis of genotype A strains circulating in Italy could certainly contribute to the definition of the source and evolution of this genotype.

Data from the present study showed that, in comparison with AHB patients infected with genotype $\mathrm{D}$, those infected with non-D genotypes were more frequently male and had higher total bilirubin level and HBV viral load at enrolment. Besides, they harbored BCP/PC mutant strains less frequently and were $\mathrm{HBeAg}$-positive more often. All these clinical and virological features have already been reported to be distinctive of AHB by genotype A in other countries $[1,11,12,26,27,30,31]$, but these characteristics have been only incompletely recognized among genotype A (or non-D) AHB patients thus far in Italy $[17,18]$. The less frequent detection of $\mathrm{PC}$ mutations in non-D genotype, most notably genotype $\mathrm{A}$, has been ascribed to the instability caused by this mutation in the stem-loop structures of the pregenome encapsidation signal $[27,32]$. This may explain the association between non-D genotype and HBsAg positivity. However, as we and others have observed, this association seems also to be independent from the BCP/PC status of patients [14].

Two patients, both harboring genotype $\mathrm{D}$, were $\mathrm{HBsAg}$ negative at enrollment. Neither of them showed seroconversion for HBsAg during the follow-up. Low levels of HBsAg in acute hepatitis B have been occasionally reported, usually in patients with low replication levels of HBV [33]. These two cases had indeed a very low viral load (1131 and $7 \mathrm{IU} / \mathrm{mL}$, respectively) on the initial sample that tested negative for HBsAg.

In this study a low rate $(2.8 \%)$ of AHB chronic evolution was found. This is in agreement with previous studies showing that in immune-competent adults HBV persistence after AHB is infrequent [1, 17-19, 34]. Several recent studies, mainly from Japan, have suggested that high viral load and chronic evolution of AHB are more frequent with genotype A infections than with infections due to other genotypes [11, 12, 26, 27, 30, 31]. Among patients investigated by Coppola et al., only three patients developed persistent infection and they all harbored genotype D [17]; whereas
Scalia et al. [18] found that the mean time to HBsAg clearance was longer when the proportion of AHB cases infected with non-D genotypes increased. We also found that HBsAg clearance was significantly delayed in patients infected with non-D genotypes who recovered from AHB, and this finding is consistent with those of other studies $[18,27,30,31]$. In our cohort two patients developed persistent infection: one with genotype A, the other with genotype $\mathrm{D}$, with an essentially similar rate of chronic evolution (3.5 vs $2.7 \%$ ), but the numbers were too small to enable any meaningful analysis.

Fulminant AHB has been reported to be associated with non-A genotypes, HBeAg-negative status and, above all, the presence of PC e BPC mutations [12, 13]. Although there were only four fulminant AHB cases in our study and $\mathrm{BCP} / \mathrm{PC}$ mutations could be assessed in three of them, it is intriguing that two patients had $\mathrm{BCP} / \mathrm{PC}$ mutations. A preexiting $\mathrm{HCV}$ chronic infection was identified as the only factor associated with a severe or fulminant course of AHB among the cohort of Italian patients investigated by Coppola et al. [19]. No coinfection with other hepatitis viruses was detected in our four patients with fulminant hepatitis and none of them reported history of chronic liver disease.

Two patients developed AHB despite previous vaccination, although G145R mutation, classically associated with vaccine escape, was not detected, suggesting that the possible selective pressure induced by universal immunization has not influenced the emergence and spread of the escape variant.

One of the two vaccine recipients suffered from insulindependent diabetes mellitus, a condition already associated with reduced efficacy of HBV vaccination in adults and children [35]. Besides, he was infected with genotype $\mathrm{F}$, which has marked sequence differences in the $\mathrm{S}$ and $\mathrm{P}$ gene compared to other genotypes. Since current vaccines are based on recombinant HBsAg protein from genotype $\mathrm{A}$ and $\mathrm{D}$, they may not result in full protective immunity towards genotype $\mathrm{F}$ infection, even in immune competent, fully vaccinated individuals with proper anti-HBs titers [36]. The second patient was infected with genotype D, yet he showed the T126I mutation in the "a" determinant of the $\mathrm{S}$ gene. This mutation may have a major impact on HBsAg antigenicity and has been associated, especially in combination with G145R mutation, with diagnosis failure (occult infections), poor disease outcome and possible escape of vaccine-induced neutralizing antibodies [37, 38]. However, since pre- and post-vaccination serological testing was not performed in any of these two patients and both of them were anti-HBs-negative at enrolment, no firm conclusion can be drawn to explain the reasons for their acute infection.

We acknowledge that the sample of AHB patients enrolled in our study was not particularly large. It is 
necessary to consider that, as consequence of universal vaccination, the incidence of symptomatic AHB at the time of the study was already very low in Italy and the vast majority of cases were aged over 25 years [6]. Nevertheless, our study population represented $12 \%$ of all the symptomatic cases reported to the Italian surveillance system for acute viral hepatitis in the same time range [6] showing similar age, sex, geographical area of residence and risk factors as those reported by the whole cohort (data not shown). Consequently, our study sample may be considered fairly representative of all the AHB cases reported in Italy in the same period. Another limitation of our study was that about $30 \%$ of patients were lost to follow-up (20\% within the first month after the enrolment). We hypothesize that the low compliance with follow-up among AHB patients was also due to the fact that they were made aware of the favorable disease outcome in most cases. Besides patients, even if still positive for HBV markers, usually feel good after hospital discharge and are probably less inclined to adhere to follow-up visits.

In conclusion, the results of our study show and confirm the increasing spread of non-D genotypes in Italy and the substantial changes in the distribution and relevance of the various risk factors for AHB. High-risk sexual exposure has a major role in HBV acquisition and is significantly associated with non-D genotype infection. HBV vaccination should be recommended and implemented among adults with high-risk sexual behavior.

Further studies on large samples of AHB patients in our geographical area are needed to enable a reliable analysis of the relationship between HBV genotypes and chronic infection, as well as the relationship between BCP/PC mutations and disease severity.

Acknowledgments The authors thank Lorenzo Fantozzi for his technical assistance. This work was supported in part by the project "Sorveglianze speciali" of the Italian Ministry of the Health (Istituto Superiore di Sanità- Fasc. ISS 1M56.) Abbott Diagnostics Italy has supported this study by providing the reagents for the serological assays and sample transportation from the clinical sites to the reference laboratories.

Conflict of interest All authors, with the exception of Claudio Galli, Alessandro R. Zanetti and Gloria Taliani, do not have potential competing interests. Claudio Galli is currently employed by Abbott Diagnostics Italy as the Scientific Affairs Manager. Abbott Diagnostics Italy has supported this study by providing the reagents for the serological assays and sample transportation from the clinical sites to the reference laboratories. Alessandro R. Zanetti has received a speaker honorarium from Glaxo Smith Kline and a consulting honorarium from Sanofi Pasteur MSD. Gloria Taliani has received speaker's honoraria form Bristol-Myers Squibb, Gilead, Janssen Cilag, Merck and AbbVie.

Ethical approval This study has been performed in accordance with the 1964 Declaration of Helsinki and its later amendments and was approved by the Istituto Superiore di Sanità ethics committee.

\section{Appendix}

Members of the Study Group include:

Enzo Raise (Department of Tropical and Infectious Disease, Ospedale dell'Angelo, Mestre, Venezia, Italy); Franklyn Esoka Eseme (Department of Tropical and Infectious Disease, Ospedale dell'Angelo, Mestre, Venezia, Italy); Giuseppe Maio (Division of Infectious Diseases, G. Rummo Hospital, Benevento, Italy); Patrizia Maio (Infectious Diseases Unit, S.G. Moscati Hospital, Avellino, Italy); Cinzia Di Giuli (Infectious Diseases Clinic, Santa Maria Hospital, Terni, Italy); Pietro Bellissima (Infectious Diseases Unit, Gravina Hospital, Caltagirone, Catania, Italy); Salvatore Bonfante (Infectious Diseases Unit, Gravina Hospital, Caltagirone, Catania, Italy); Piergiorgio Chiriacò (Infectious Diseases Unit, A. Perrino Hospital, Brindisi, Italy); Bruno Cacopardo (Department of Clinical and Molecular Biomedicine, Division of Infectious Diseases, University of Catania, Italy); Pierluigi Blanc (Infectious Diseases Unit, Malattie infettive. S. Maria Annunziata Hospital, Firenze, Italy); Stefania Palattella (Clinic of Infectious Diseases, University of Bari, Bari, Italy); Teresa Santantonio (Clinic of Infectious Diseases, University of Bari, Bari, Italy); Angela Guastadisegni (Clinic of Infectious Diseases, University of Bari, Bari, Italy); Pietro Amoroso (Department of Infectious Diseases, Cotugno Hospital, Naples, Italy); Paolo Fabris (Department of Infectious Diseases and Tropical Medicine, S. Bortolo Hospital, Vicenza, Italy); Maria Teresa Giordani (Department of Infectious Diseases and Tropical Medicine, S. Bortolo Hospital, Vicenza, Italy); Vincenzo Vullo (Department of Public Health and Infectious Diseases, University of Rome "Sapienza"Rome Italy), Claudia D'Agostino (Department of Public Health and Infectious Diseases,University of Rome "Sapienza"Rome Italy); Tiziana Giuberti (Unit of Infectious Diseases and Hepatology, Azienda OspedalieroUniversitaria di Parma, Parma, Italy); Simona Schivazzappa (Unit of Infectious Diseases and Hepatology, Azienda Ospedaliero-Universitaria di Parma, Parma, Italy); Michele Trezzi (Division of Infectious Diseases, "Santa Maria della Misericordia" Hospital, Grosseto, Italy); Gabriella Verucchi (Infectious Diseases Section, Department of Internal Medicine, Geriatric Medicine and Nephrology, University of Bologna, Bologna, Italy); Carlo Biagetti (Infectious Diseases Section, Department of Internal Medicine, Geriatric Medicine and Nephrology, University of Bologna, Bologna, Italy); Daria Pocaterra (Infectious Diseases Section, Department of Internal Medicine, Geriatric Medicine and Nephrology, University of Bologna, Bologna, Italy); Evangelista Sagnelli (Department of Public Medicine, Second University of Naples, Naples, Italy); Vincenzo Messina (Infectious Diseases Unit, A.O.R.N. Sant'Anna e San Sebastiano, Caserta, Italy); Giorgio Antonucci (Clinical 
Department of Infectious Diseases, National Institute for Infectious Disease, L. Spallanzani, Rome, Italy); Francesco Vairo (Clinical Department of Infectious Diseases, National Institute for Infectious Disease, L. Spallanzani, Rome, Italy); Marcello Marinelli, (Microbiology, IRCCS San Raffaele, Milan, Italy); Sara Paladini, Catia Tagliacarne (University of Milan, Department of Biomedical Sciences for Health, Milan, Italy).

\section{References}

1. Liaw YF, Chu CM. Hepatitis B virus infection. Lancet. 2009;373:582-92.

2. World Health Organization (WHO). Available, from: http://www. who.int/mediacentre/factsheets/fs204/en/. Updated April 2014.

3. Romanò L, Velati C, Cambiè G, et al. Hepatitis B virus infection among first-time blood donors in Italy: prevalence and correlates between serological patterns and occult infection. Blood Transfus. $2013 ; 1: 281-8$.

4. Stroffolini T, Guadagnino V, Rapicetta M, et al. The impact of a vaccination campaign against hepatitis $B$ on the further decrease of hepatitis B virus infection in a southern Italian town over 14 years. Eur J Intern Med. 2012;23:e190-2.

5. Fabris P, Baldo V, Baldovin T, et al. Changing epidemiology of $\mathrm{HCV}$ and HBV infections in Northern Italy: a survey in the general population. J Clin Gastroenterol. 2008;42:527-32.

6. Integrated epidemiological system for acute viral hepatitis. Available, from: http://www.iss.it/seieva/. Updated April 2014.

7. Mele A, Tosti ME, Mariano A, et al. Acute hepatitis B 14 years after the implementation of universal vaccination in Italy: areas of improvement and emerging challenges. Clin Infect Dis. 2008;46:868-75.

8. Palumbo E, Scotto G, Cibelli DC, et al. Immigration and hepatitis B virus: epidemiological, clinical and therapeutic aspects. East Mediterr Health J. 2008;14:784-90.

9. Fasano M, Saracino A, Carosi G, et al. Hepatitis B and immigrants: a SIMIT multicenter cross-sectional study. Infection. 2013;41:53-9.

10. Shi YH. Correlation between hepatitis B virus genotypes and clinical outcomes. Jpn J Infect Dis. 2012;65:476-82.

11. Lin CL, Kao JH. The clinical implications of hepatitis B virus genotype: recent advances. J Gastroenterol Hepatol. 2011;26:123-30.

12. Ozasa A, Tanaka Y, Orito E, et al. Influence of genotypes and precore mutations on fulminant or chronic outcome of acute hepatitis B virus infection. Hepatology. 2006;44:326-34.

13. Ehata T, Omata M, Chuang WL, et al. Mutations in core nucleotide sequence of hepatitis B virus correlate with fulminant and severe hepatitis. J Clin Invest. 1993;91:1206-13.

14. Dal Molin G, Poli A, Crocè LS, D'Agaro P, et al. Hepatitis B virus genotypes, core promoter variants, and precore stop codon variants in patients infected chronically in North-Eastern Italy. J Med Virol. 2006;78:734-40.

15. Brunetto MR, Giarin MM, Oliveri F, et al. Wild-type and e antigen-minus hepatitis B viruses and course of chronic hepatitis. Proc Natl Acad Sci USA. 1991;88:4186-90.

16. De Mitri MS, Cassini R, Morsica G, et al. Virological analysis, genotypes and mutational patterns of the HBV precore/core gene in HBV/HCV-related hepatocellular carcinoma. J Viral Hepat. 2006;13:574-81.
17. Coppola N, Masiello A, Tonziello G, et al. Factors affecting the changes in molecular epidemiology of acute hepatitis B in a Southern Italian area. J Viral Hepat. 2010;17:493-500.

18. Scalia G, Russo R, Palermo I, et al. Clinical, virological and epidemiological aspects of acute B hepatitis in the Catania area (Italy). Infez Med. 2010;18:169-74.

19. Coppola N, Sagnelli C, Pisaturo M, et al. Clinical and virological characteristics associated with severe acute hepatitis B. Clin Microbiol Infect. 2014;20:0991-7. doi:10.1111/1469-0691.12720.

20. Stroffolini T, Rapicetta M, Lombardo F, et al. Historical study of acute hepatitis B in subjects with or without hepatitis $\mathrm{C}$ infection. Eur J Intern Med. 2012;23:e146-9.

21. Rodella A, Galli C, Terlenghi L, et al. Quantitative analysis of $\mathrm{HBsAg}, \mathrm{IgM}$ anti-HBc and anti-HBc avidity in acute and chronic hepatitis B. J Clin Virol. 2006;37:206-12.

22. Chan HL, Hussain M, Lok AS. Different hepatitis B virus genotypes are associated with different mutations in the core promoter and precore regions during hepatitis $\mathrm{B}$ e antigen seroconversion. Hepatology. 1999;29:976-84.

23. Chemin I, Zoulim F, Merle P, et al. High incidence of hepatitis B infections among chronic hepatitis cases of unknown aetiology. $\mathrm{J}$ Hepatol. 2001;34:447-54.

24. Sagnelli C, Ciccozzi M, Pisaturo M, et al. Molecular epidemiology of hepatitis B virus genotypes circulating in acute hepatitis B patients in the Campania region. J Med Virol. 2014;86: 1683-93.

25. Kobayashi M, Ikeda K, Arase Y, et al. Change of hepatitis B virus genotypes in acute and chronic infections in Japan. J Med Virol. 2008;80:1880-4.

26. Tamada Y, Yatsuhashi H, Masaki N, et al. Hepatitis B virus strains of subgenotype A2 with an identical sequence spreading rapidly from the capital region to all over Japan in patients with acute hepatitis B. Gut. 2012;61:765-73.

27. Yotsuyanagi H, Okuse C, Yasuda K, et al. Distinct geographic distributions of hepatitis B virus genotypes in patients with acute infection in Japan. J Med Virol. 2005;77:39-46.

28. van Houdt R, Bruisten SM, Koedijk FD, et al. Molecular epidemiology of acute hepatitis B in the Netherlands in 2004: nationwide survey. J Med Virol. 2007;79:895-901.

29. Sloan RD, Strang AL, Ramsay ME, et al. Genotyping of acute HBV isolates from England, 1997-2001. J Clin Virol. 2009;44:157-60.

30. Yotsuyanagi H, Ito K, Yamada N, et al. High levels of hepatitis $\mathrm{B}$ virus after the onset of disease lead to chronic infection in patients with acute hepatitis B. Clin Infect Dis. 2013;57: 935-42.

31. Ito K, Yotsuyanagi H, Yatsuhashi H, et al. Risk factors for longterm persistence of serum hepatitis B surface antigen following acute hepatitis B virus infection in Japanese adults. Hepatology. 2014;59:89-97.

32. Lok AS, Akarca U, Greene S. Mutations in the pre-core region of hepatitis B virus serve to enhance the stability of the secondary structure of the pre-genome encapsidation signal. Proc Natl Acad Sci USA. 1994;91:4077-81.

33. Chulanov VP, Shipulin GA, Schaefer S, et al. Kinetics of HBV DNA and HBsAg in acute hepatitis B patients with and without coinfection by other hepatitis viruses. J Med Virol. 2003;69:313-23.

34. Hyams KC. Risks of chronicity following acute hepatitis B virus infection: a review. Clin Infect Dis. 1995;20:992-1000.

35. Schillie SF, Spradling PR, Murphy TV. Immune response of hepatitis B vaccine among persons with diabetes: a systematic review of the literature. Diabetes Care. 2012;35:2690-7. 
36. Tacke F, Amini-Bavil-Olyaee S, Heim A, et al. Acute hepatitis B virus infection by genotype $\mathrm{F}$ despite successful vaccination in an immune-competent German patient. J Clin Virol. 2007;38:353-7.

37. Ren F, Tsubota A, Hirokawa T, et al. A unique amino acid substitution, T126I, in human genotype $\mathrm{C}$ of hepatitis B virus $\mathrm{S}$ gene and its possible influence on antigenic structural change. Gene. 2006;383:43-51.
38. Sticchi L, Caligiuri P, Cacciani R, et al. Epidemiology of HBV S-gene mutants in the Liguria Region, Italy: implications for surveillance and detection of new escape variants. Hum Vaccin Immunother. 2013;9:568-71. 